\title{
Advances in the care of children with lupus nephritis
}

\author{
Scott E. Wenderfer', Natasha M. Ruth ${ }^{2}$ and Hermine I. Brunner ${ }^{3}$
}

The care of children with lupus nephritis (LN) has changed dramatically over the past $50 \mathrm{y}$. The majority of patients with childhood-onset systemic lupus erythematosus (CSLE) develop LN. In the 1960's, prognosis in children was worse than in adults; therapies were limited and toxic. Nearly half of cases resulted in death within $2 \mathrm{y}$. Since this time, several diagnostic recommendations and disease-specific indices have been developed to assist physicians caring for patients with LN. Pediatric researchers are validating and adapting these indices and guidelines for the treatment of LN in CSLE. Classification systems, activity, and chronicity indices for kidney biopsy have been validated in pediatric cohorts in several countries. Implementation of contemporary immunosuppressive agents has reduced treatment toxicity and improved outcomes. Biomarkers sensitive to LN in children have been identified in the kidney, urine, and blood. Multi-institutional collaborative networks have formed to address the challenges of pediatric LN research. Considerable variation in evaluation and treatment has been addressed for proliferative forms of $\mathrm{LN}$ by development of consensus treatment practices. Patient survival at $5 \mathrm{y}$ is now 95-97\% and renal survival exceeds 90\%. Moreover, international consensus exists for quality indicators for CSLE that consider the unique aspects of chronic disease in childhood.

S ystemic lupus erythematosus (SLE) is an autoimmune inflammatory disease characterized by antibodies directed against self-antigens, resulting in multi-organ damage. In the United States, SLE primarily affects young women of non-white ancestry. Up to $20 \%$ of cases are diagnosed during childhood, i.e., disease onset prior to age $18 \mathrm{y}$. Differences in reported prevalence rates of childhood-onset disease (cSLE) stem from racial variations of the populations reported as well as various definitions for cSLE. There has been variability in age cut-offs used to define cSLE, ranging from 14 to $21 \mathrm{y}(1)$.

Between 40 and $70 \%$ of cSLE patients will develop kidney involvement (lupus nephritis (LN)), during their disease course (2-4), and a meta-analysis shows a $10-30 \%$ higher prevalence in cSLE than in adult-onset SLE (2). In children, LN tends to present earlier and behaves more aggressively $(4,5)$.

In the late 1950's, death from SLE within the first 2 y after diagnosis was common and available therapies were highly toxic (6-8). Mortality due to active disease was matched by mortality related to the adverse effects from immunosuppression. Fortunately, there have been significant advancements in the management of adult-onset and cSLE, resulting in dramatic improvements in short- and long-term patient and renal survival. By reviewing the numerous studies published by pediatric researchers on the management of LN in CSLE, we highlight the advancements over the past $50 \mathrm{y}$.

\section{ADVANCES IN DIAGNOSIS}

SLE manifests differently in each individual patient. Therefore, making the diagnosis can be a challenge. Discovered in 1948, the first diagnostic marker for the identification of SLE was the so-called lupus erythematosus cell (9), a phagocyte which has engulfed the denatured nuclear material of another cell. This was followed by the discovery of anti-nuclear antibodies (ANA) in SLE patients and later the devlopment of a panel of ANA which included antibodies against double-stranded DNA, RNA, and specific ribonuclear proteins.

However, the presence of ANA is not specific for lupus, and a positive test in isolation is not sufficient to make the diagnosis of SLE. A major advance towards studying and managing lupus came with the development of the American College of Rheumatology (ACR) classification criteria for SLE (10). Although intended to limit variability in the recruitment of individuals for SLE research, physicians have adapted the ACR criteria to assist with SLE diagnosis. Table 1 includes an abbreviated list of the 11 classification criteria. For a diagnosis of SLE to be made, individuals must develop disease manifestations meeting the classification criteria in at least four areas. Therefore, an elevated level of ANA is more appropriately supportive of a clinical suspicion of for lupus individuals who present with a history of three or more other classification criteria.

The ACR classification criteria have been successfully applied to CSLE $(5,11)$. Given that renal involvement can precede serological and extra-renal manifestations, delaying targeted treatment, the Systemic Lupus International Collaborating Clinics criteria were developed to allow for diagnosis of SLE with only biopsy proven lupus nephritis (12). However, the Systemic Lupus International Collaborating Clinics criteria

\footnotetext{
'Department of Pediatrics, Baylor College of Medicine, and Renal Section, Texas Children's Hospital, Houston, Texas; ${ }^{2}$ Pediatric Rheumatology, Medical University of South Carolina Children's Hospital, Charleston, South Carolina; ${ }^{3}$ Division of Rheumatology, Cincinnati Children's Hospital Medical Center, Department of Pediatrics, University of Cincinnati, Cincinnati, Ohio. Correspondence: Scott E. Wenderfer (sewender@texaschildrens.org)

Received 4 August 2016; accepted 7 October 2016; advance online publication 4 January 2017. doi:10.1038/pr.2016.247
} 
Table 1. American College of Rheumatology classification criteria for systemic lupus erythematosus

\begin{tabular}{l} 
1. Malar rash \\
2. Discoid rash \\
3. Photosensitivity \\
4. Oral ulcers \\
5. Serositis (Pericarditis or pleuritis) \\
6. Arthritis \\
7. Kidney disease (nephritis or glomerulopathy) \\
8. Neurologic disease (seizure or psychosis) \\
9. Hematologic disease (autoimmune cytopenias) \\
10. Immunologic (anti-DNA, anti-RNP, aPL antibodies) \\
11. Antinuclear antibodies \\
\hline aRNP = ribo-nuclear protein, aPL = anti-phospholipid.
\end{tabular}

have markedly lower specificity for adult or $\operatorname{cSLE}(13,14)$ and have not been endorsed by the ACR.

\section{ADVANCES IN INTERPRETATION OF KIDNEY BIOPSIES FOR LUPUS NEPHRITIS}

Kidney biopsy remains the gold standard for diagnosis of LN in cSLE. In children, as in adults, the procedure is performed percutaneously with ultrasound guidance. Kidney biopsy requires the involvement of several medical teams and prolonged observation post procedure, and is a source of emotional distress for the family and the child. Unlike adults who receive only local anesthesia, children often have the procedure performed under conscious sedation or even general anesthesia. Typically, two tissue cores are obtained via 16- or 18-gauge needles. The increased availability of procedural imaging and automated needles has reduced adverse events (15). Registry studies from Norway support that kidney biopsies are as safe in children as in adults: $1.7 \%$ of 715 children developed gross hematuria, $0.1 \%$ required blood transfusion, and $0.1 \%$ required surgery for vascular complications (16).

The first classification system for LN (the World Health Organization classification, developed in the 1970s) was superseded by the revised classification of the International Society of Nephrology/Renal Pathology Society (ISN/RPS) classification (17) (Table 2). Accurate diagnosis requires at least 10 glomeruli to be present in the biopsy tissue in order to reasonably exclude focal lesions and ensure a proper characterization of kidney involvement (18). The occurrence of the classes of LN in adults and children at new diagnosis is identical (19). The utility of distinguishing between histological classes of LN, such as proliferative and membranous types, and between segmental and global forms of proliferative $\mathrm{LN}$ is supported by studies of cSLE (20-23).

The ISN/RPS classification improved the precision of class definitions and distinguished between active and chronic lesions (17), although inter-pathologist variation and reproducibility remain suboptimal. Active lesions are amenable to immunosuppressant therapy, while chronic lesions represent nonreversible damage (24), often requiring supportive therapy
Table 2. Histological classes of lupus nephritis ${ }^{a}$

Class I: minimal mesangial
Class II: mesangioproliferative
Class III (A): focal proliferative ${ }^{b}$
Class III (C): focal proliferative ${ }^{b}$
Class III (A/C): focal proliferative
Class IV-S (A): diffuse proliferative ${ }^{b}$
Class IV-S (C): diffuse proliferative
Class IV-S (A/C): diffuse proliferative
Class IV-G (A): diffuse proliferative ${ }^{b}$
Class IV-G (C): diffuse proliferative
Class IV-G (A/C): diffuse proliferative

Class V: membranous

Class Vl: advanced sclerosing

aClassification of the International Society of Nephrology and Renal Pathology Society (17). ${ }^{\mathrm{B}} \mathrm{A}=$ active, $\mathrm{C}=$ chronic, $\mathrm{S}=$ segmental, $\mathrm{G}=$ global.

instead. Activity index (AI) and chronicity index (CI) quantify mainly glomerular injury, and tubulointerstitial activity index (TIAI) quantifies extra-glomerular kidney disease (25). As in adults, risk factors for poor outcome in cSLE include $\mathrm{AI} \geq 7$, CI $\geq 4$, and TIAI $>5(26,27)$.

Practice patterns for initial and repeat kidney biopsy have been published for cSLE. There is more disparity among either nephrologists or rheumatologists than between the two specialties (28). Most specialists use the indications for initial kidney biopsy developed by the American College of Rheumatology, whereas others use more inclusive indications and fewer only recommend biopsy when the diagnosis of cSLE is unclear. When patients with proliferative LN fail to achieve a complete clinical response upon completion of induction therapy, nearly $25 \%$ of both pediatric nephrologists and rheumatologists recommend repeat kidney biopsy to guide subsequent maintenance therapy. Far fewer pediatric rheumatologists and nephrologists perform repeat biopsy after sustained remission to support their decision to withdraw immunosuppression (28).

\section{ADVANCES IN IDENTIFICATION OF BIOMARKERS FOR NEPHRITIS}

Biomarkers are factors that can be objectively measured and used either in support of a diagnosis of LN or to predict its course and response to therapy. Biomarker studies in cSLE have been hindered by both lack of normal age-specific profiles for given substances and the relative immaturity of renal excretory capacity. In recent years, the availability of powerful tools to scan both the genome and proteome have revolutionized and greatly accelerated biomarker discovery. Pediatrician scientists have embraced these tools for the study of cSLE (29-34).

Three of the following clinical tests have been used routinely as noninvasive predictors for LN: (i) kidney function, using serum creatinine as a surrogate measure of glomerular filtration rate; (ii) urinary protein excretion; and (iii) glomerular 
hematuria, using analysis of urinary sediment (35). Although these three markers have been used to define renal response to therapy and to predict disease flares (36), they can be imprecise, and treatment decisions often require kidney biopsy.

Titers of antibodies specific for double stranded DNA can predict with modest precision the presence of LN (sensitivity $57 \%$, specificity 97\%) (37). Hypocomplementemia has also proven to be useful (64\% sensitive, 91\% specific) (38). However, hypocomplementemia and anti-dsDNA antibodies accompany SLE flares in only 54 and $27 \%$ of patients, respectively (30).

Fortunately, research in cSLE has yielded several promising biomarkers (Table 3). High urinary NGAL (neutrophil gelatinase-associated lipocalin) levels can predict disease activity and injury in cSLE with LN (29,31), and can predict renal flares with a higher sensitivity and specificity than dsDNA antibodies $(31,32)$. MCP-1 (monocyte chemoattractant protein) urinary levels can also predict improvement of renal disease (31). Despite high sensitivity and responsivity to LN activity, MCP1, RANTES, and TWEAK lack specificity for LN, and have also been found in cerebral spinal fluid and linked to the development of central neuropsychiatric involvement in cSLE (33). NGAL is also a biomarker for acute kidney injury and MCP is for chronic kidney disease in patients without SLE.

Given the diversity of LN histological features, it is unlikely that any one noninvasive biomarkers will be sufficient for monitoring LN disease activity in cSLE. However, promising

Table 3. Candidate biomarkers for lupus nephritis ${ }^{\mathrm{a}}$

\begin{tabular}{l}
\hline Serum \\
\hline Anti-C1q antibodies \\
Anti-GBM antibodies \\
APRIL \\
BAFF \\
\hline Urine \\
\hline Adiponectin \\
CCL2/MCP-1 \\
CCL5/RANTES \\
Ceruloplasmin \\
Hemopexin \\
IP-10 \\
KIM-1 \\
L-PDGS \\
NGAL \\
Orosomucoid \\
Transferrin \\
TWEAK \\
VCAM-1 \\
\hline aAPRIL, a proliferation-inducing ligand; BAFF, B-lymphocyte activating factor; CCL, \\
CC-type chemokine ligand; GBM, glomerular basement membrane; I, interferon \\
-induced protein; L-PDGS, lipocalin-type prostaglandin D synthase; KIM, kidney injury \\
molecule; RANTES, regulated on activation, normal T cell expressed and secreted; \\
TWEAK, tumor necrosis factor-like weak inducer of apoptosis; VCAM, vascular cell \\
adhesion molecule. \\
\end{tabular}

findings have been reported identifying signatures or panels of markers for LN in cSLE $(27,30)$. Discovery microarrays can be used to screen for messenger RNA (mRNA) levels. Posttranslational modifications such as glycosylation and methylation, and even disease-specific protein fragmentation, are assessed using proteomic techniques. One panel includes transferrin, orosomucoid, ceruloplasmin, and lipocalin-type prostaglandin D synthase ( $\beta$-trace protein) (30). All four proteins were found at significantly higher levels in active LN compared to nonrenal SLE or JIA controls. In urine, concentrations are increased 3 mo before renal flare. An overlapping panel of six urinary biomarkers (NGAL, MCP-1, ceruloplasmin, adiponectin, hemopexin, and kidney injury molecule-1) was found in CSLE patients to predict both AI and TIAI on kidney biopsy (27). Real-time polymerase chain reaction has also been used to assess the utility of candidate noncoding microRNAs in the urine of cSLE patients (34).

More research is warranted to identify and validate noninvasive biomarkers for monitoring disease activity. Comorbid conditions, such as hypertension or diabetes, can alter the excretion of potential biomarkers in the absence of histologic changes. However, it does not appear that different biomarker panels will be necessary for adult-onset SLE and cSLE (manuscript under review).

\section{ADVANCES IN IMMUNOSUPPRESSIVE THERAPY FOR LUPUS NEPHRITIS}

Therapeutic goals for the treatment of LN include: achieving prompt renal remission, avoiding renal flares, preventing chronic renal impairment, and minimizing iatrogenic effects. Treatment typically includes induction therapy, aimed at achieving LN remission by means of intensive immunosuppression, followed by maintenance therapy, aimed at avoiding LN flares with less intensive immunosuppression (Table 4). Responses may differ by race and ethnicity, and treatment decisions are mostly based on either the large clinical trials adult studies or small pediatric cohort studies (39). There are no established steroid-free protocols developed for CSLE.

Fifty years ago, the only pharmacologic therapy available for treating children with LN was corticosteroids (6). High-dose oral $(2 \mathrm{mg} / \mathrm{kg} / \mathrm{d})$ and intermittent "pulse" IV doses $(30 \mathrm{mg} / \mathrm{kg})$ were moderately effective, but inhibition of growth in children was concerning (40). Fortunately, several steroid-sparing therapies have been implemented. Starting in the 1970s, monthly IV dosing of cyclophosphamide (CYC) was the treatment of choice for proliferative LN and in the mid-1990s IV CYC plus pulse steroids was shown to be superior to pulse steroids alone as induction therapy. This became known as the "NIH protocol." Efficacy of comparable CYC protocols has been reported in pediatric cohorts (41-43). However, high rates of gonadal toxicity, serious infection, and malignancy with this regimen are a concern. A lower dose CYC protocol was initially used in Europe in adults (the "Euro-lupus protocol") (44) and holds promise for $\mathrm{LN}$ in children.

Another less toxic approach to induction therapy has been mycophenolate mofetil (MMF), an inhibitor of the de novo 
Table 4. Pharmacotherapy for childhood-onset SLE

\begin{tabular}{l} 
Induction/initial therapy \\
Corticosteroids ${ }^{\mathrm{a}}$ \\
Cyclophosphamide \\
Mycophenolate mofetil \\
Secondary for refractory disease \\
Rituximab \\
Maintenance therapy \\
Mycophenolate mofetil \\
Azathioprine \\
Hydroxychloroquine ${ }^{\mathrm{a}}$ \\
Chloroquine \\
Anticoagulation therapy \\
Aspirin \\
Coumadin \\
Low-molecular weight heparin \\
Renin Angiotensin Blockade \\
Angiotensin converting enzyme inhibitors \\
Angiotensin receptor blockers \\
Diuretic therapy \\
Loop diuretics \\
\hline Approved by the US Food and Drug Administration for SLE
\end{tabular}

${ }^{a}$ Approved by the US Food and Drug Administration for SLE.

purine synthesis pathway with selectivity for proliferating lymphocytes. Following several non-inferiority studies in adult LN patients, retrospective studies have confirmed efficacy as induction $(42,45)$ and maintenance therapy $(46)$, as well as for rescue therapy of refractory LN (47) in children. Small pediatric studies have reported efficacy for MMF for class II (45), III (42), and V LN (47).

Other immunosuppressive agents with some evidence for efficacy in LN include: azathioprine (AZA), tacrolimus (TAC), and cyclosporine (CSA). Retrospective data in children showed a favorable response to AZA and prednisone compared to CYC and prednisone for induction therapy of $\mathrm{LN}$ (43). The MAINTAIN trial reported good efficacy for either AZA or MMF for maintenance therapy of LN in adults (48). A multi-center, randomized controlled study of 81 subjects as young as $14 \mathrm{y}$ of age with $\mathrm{LN}$ in China suggests comparable renal response rates $(90 \%)$ and superior complete response rates (52\%) using TAC plus prednisone versus IV CYC plus prednisone (82 and 39\%) (49). Adverse events were less frequent (GI, leukopenia) with TAC. A prospective randomized trial showed comparable outcomes between CSA and CYC in children (50). Retrospective data in children support the efficacy of sequential induction therapy with MMF followed by CSA for proliferative LN (51).

Despite prospective clinical trials failing to show benefit for use of B-cell depleting agents in SLE, there are numerous observational studies reporting efficacy for refractory disease using rituximab as an add-on therapy for use in both adultand CSLE (52-56). A UK pediatric cohort study (25 patients with LN, 38 courses) showed improved disease activity (55). The safety of rituximab use for pediatric autoimmune diseases has been assessed in a single center study of 104 patients (including 50 with cSLE) and the rate of infections requiring hospitalization was $9.1 \%$ (56).

Since conducting large-scale clinical trials in cSLE is not feasible, due to small population size and lack of funding, reduction of clinical practice variability through the development of consensus treatment plans (CTPs) is an alternative approach that provides for future comparison of outcomes and standardization of therapy. The development of CTPs in 2012 for induction therapy of newly diagnosed proliferative LN in cSLE represents a tremendous advancement (36). The CTPs provide three strategies for standardized use of glucocorticoids, including primarily oral, mixed oral/IV and primarily IV regimens. CTPs are also included for initial therapy with either daily oral MMF or monthly IV CYC for 6 mo. Research studies are ongoing to gauge the utility of these CTPs at individual pediatric sites. Consistent use of the CTPs may improve the prognosis of proliferative LN and will facilitate the conduct of future comparative effectiveness studies aimed at optimizing therapeutic strategies.

\section{TOOLS TO MONITOR SLE AND ADVANCES IN CHRONIC DISEASE MANAGEMENT}

Managing chronic illness is time consuming and complicated. Scoring indices that track treatable disease activity and nonreversible damage have been developed for lupus (57). Single center studies from Canada and Brazil have validated the utility of these activity and damage indices in cSLE patients $(58,59)$, but modifications of the scales developed for adults with SLE have improved overall accuracy (60). Outcomes research in cSLE has also evolved to include health-related quality of life (HRQOL). High-quality HRQOL data for cSLE patients from four large centers in Canada (61) has provided a baseline for comparing disease interventions with respect to disease activity and accumulated damage.

Outcomes depended on numerous factors including: patient and family resources, physician resources, and the medical institution's size and commitment to quality care. It is important for each medical visit to address not only acute problems, but also health maintenance. Important collaborative multicenter studies have addressed some of these issues in cSLE, including a randomized double-blind placebo-controlled clinical trial of routine statin use in subclinical atherosclerosis progression (62).

There has also been a move toward quality driven care. In 2001, The US Institute of Medicine issued a report citing safety deficiencies in the American health care system stemming from a lack of metrics to assess the quality of patient care (63). In response, an international consensus was reached for a set of process quality indicators for cSLE (Table 5) (64). Assessment between 2011-2014 shows that these quality indicators had not been consistently met (65) and likely contributed to suboptimal clinical outcomes. Further studies will address the needs of patients, families, physicians, and medical institutions 
in order to meet these minimal standards. Learning networks have been developed to address quality improvement approaches in complex, multicenter health care systems. One example is PR-COIN (Pediatric Rheumatology Collaborative Improvement Network), a learning-collaborative including over a dozen pediatric rheumatology sites (https://pr-coin. $\operatorname{org} /)$.

Another advance in our approach to better management of cSLE has come from involving our patients and their families in clinical research projects (66). Such "patient oriented research" has begun by using focus groups and has identified problems that cSLE patients themselves want addressed: marred identity, restricted major life decisions, uncertainty regarding their health, resentment of long-term treatment, and lack of resilience. These studies allow for more focused needs based psychosocial and educational interventions.

The availability of resources to treat cSLE patients and improve access of care is of utmost importance. International consensus has been reached on preliminary criteria for diagnosing global flares in cSLE (67). Comprehensive care of a pediatric patient with SLE requires a multidisciplinary team including: pediatric rheumatology, nephrology, ophthalmology, psychology, the primary care physician and/or adolescent medicine, and sometimes physical therapy, dermatology, cardiology, orthopedics, neurology, gastroenterology, pulmonary, or infectious disease. Patients and families need to have social workers available as well. This becomes most important at the time of transition to adult care providers. Transition programs and access to care teams in the adult world allows for a smoother transition for patients with chronic disease, and research has been published specifically for childhood-onset SLE patients (68). Many institutions have moved towards comprehensive clinics that include appointments with pediatric rheumatology and pediatric nephrology as well as adolescent medicine. In the future, these multidisciplinary teams may grow to include access to more specialists.

Table 5. International consensus quality indicators for childhoodonset SLE

1. Use of daily sunscreen

2. Eye screening on anti-malarials

3. Daily exercise to help prevent cardiovascular disease

4. Routine laboratory screen for lupus activity

5. Reproductive health discussions including birth control and STDs

6. Bone health and the need for both calcium and vitamin D

7. Management of blood pressure and proteinuria with ACEi or ARBs

8. Assessment for influenza, pneumococcal, and meningococcal vaccinations

9. Assessment for changes in cognitive performance at school or in the home

aCEi, angiotensin converting enzyme inhibitors; ARBs, angiotensin receptor blockers; STD, sexually transmitted diseases.

\section{ADVANCES IN INFRASTRUCTURE TO STUDY LN IN CHILDREN}

There have been several advances toward the goal of implementing best practices in cSLE. Ideally, a patient who develops disease in a small town will receive the same care as another child diagnosed in a large medical center across town or across the globe. Evidence-based guidelines have not been available for children with SLE and this has not changed in the past 50 y. Due to small sample sizes and limited funding for research, many management decisions made caring for CSLE patients will never be truly evidence based. Chapter 12, section 12 of the KDIGO (Kidney Disease Improving Global Outcomes) guidelines for glomerulonephritis suggest that children with $\mathrm{LN}$ receive the same therapies as adults with $\mathrm{LN}$, with dosing based on patient size and GFR (69). This is because the quality of evidence for a pediatric-specific approach to $\mathrm{LN}$ was deemed very low.

Disease registries help in assessing patient outcomes especially in regards to treatment. The Childhood Arthritis Rheumatology Research Alliance (CARRA) registry includes an observational longitudinal data capture resource from clinical sites representing all major geographic regions of the United States (19). The Italian Collaborative Study (70) and the 1000 Canadian Faces of Lupus Cohort (71) both provided useful information as well. In addition, cohorts such as the UK JSLE Cohort Study $(4,23,31,55)$, the PULSE cohort in Africa (72), and the Israeli National Registry of Children with Rheumatic Diseases (73) are actively enrolling pediatric lupus patients elsewhere around the world. The Pediatric Rheumatology International Trials Organization (PRINTO) and the Pediatric Rheumatology Collaborative Study Group are two research networks that specialize in conducting studies in pediatric rheumatology, including cSLE. The Midwest Pediatric Nephrology Consortium (MWPNC) maintains an active registry (manuscript in preparation) as do several pediatric hospitals and institutions. In North America, the Pediatric Nephrology and Rheumatology Collaborative Group (PNR-CG) was established in 2014 with a goal of promoting multi-disciplinary research into cSLE and developing more consensus treatment plans. The first initiative of this group resulted in a publication on practice patterns for kidney biopsy in cSLE (28). Consensus building nationally and internationally will allow for fewer confounders when assessing retrospective data and will aid in the design of prospective clinical trials.

\section{OUTCOMES OF LUPUS NEPHRITIS IN CHILDREN IN 2016}

Renal involvement in SLE increases morbidity due to the effects of high-dose immunosuppression, renal dysfunction, and hypertension on the brain, cardiovascular system, and the bones during growth and development. Although they differ based on ethnicity, race, and socioeconomic status, outcomes have greatly improved over the past several decades (Table 6). Prior to corticosteroid therapy, patient survival did not exceed $5 \mathrm{y}(6,7)$. At that time, progression to end-stage kidney disease (ESKD) had a high mortality rate in children. One third died from complications of kidney failure, another third died of 
Table 6. Outcomes of lupus nephritis in cSLE over the past $50 \mathrm{y}^{\mathrm{a}}$

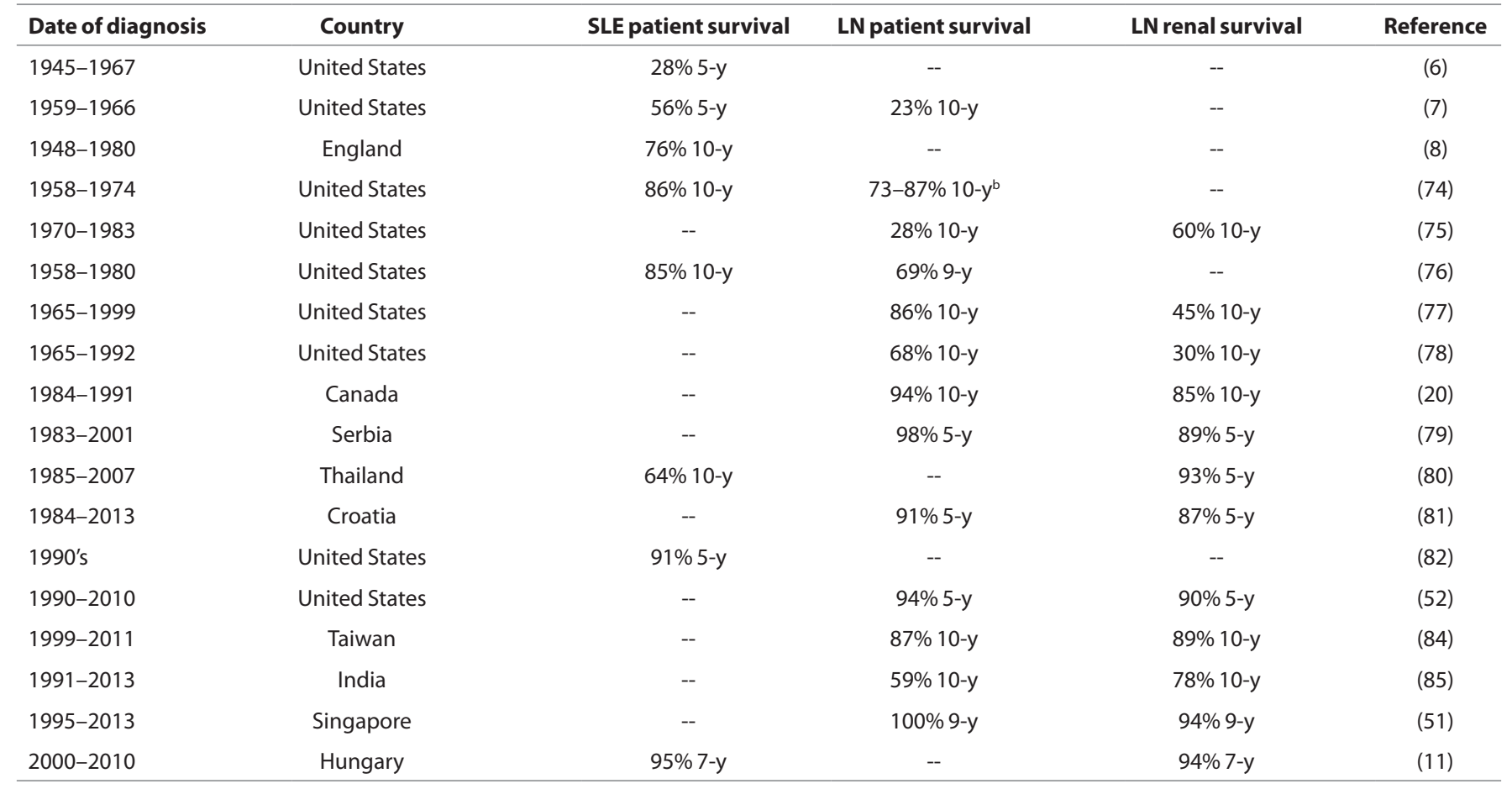

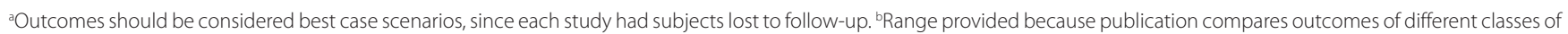
lupus nephritis

sepsis or severe infections, whereas CNS vasculitis and pulmonary hemorrhage played a more minor role. Outcomes greatly improved by 1990 in both children $(52,74-82)$ and adults (83) with SLE and LN (92-95\% patient and $89-90 \%$ renal survival $10 \mathrm{y}$ after diagnosis), but have been unchanged over the past 2 decades $(52,80-85)$. In the 21 st century, the main causes of death in SLE are cardiopulmonary and infectious. The management change associated temporally with the largest improvement in renal survival was the addition of maintenance immunosuppression after induction therapy (52).

Despite immunosuppression, only 55\% of cSLE patients with proliferative LN (class III and IV) achieve renal remission $(22,86,87)$. While $90 \%$ of cSLE patients with class V LN in cSLE achieve renal remission, only $76 \%$ can maintain remission despite low dose oral corticosteroids and/or maintenance immunosuppression such as AZA or MMF $(88,89)$. The rate of kidney flares due to SLE is $25-50 \%$ on therapy $(51,84,89)$. Besides class IV LN, risk factors for development of ESKD include male gender, black race, hypertension, nephrotic syndrome, anti-phospholipid antibodies, high glomerular staining for MCP-1, chronicity on biopsy, poor response to induction therapy, and occurrence of nephritic kidney flare $(23,87)$.

The mortality rate on dialysis $(22 \%$ at $5 \mathrm{y})$ is similar to that reported for other causes of pediatric-onset ESKD (90). One third of cSLE patients with LN and ESKD receive a kidney transplant within $5 \mathrm{y}$. Based on data from the US Renal Data System (USRDS) from 1995 to 2006, 51\% were African American and 24\% Hispanic (90). There were fewer kidney transplants among older vs. younger (odds ratio (OR): 0.59, confidence interval (CI): 0.43-0.81), African American vs. white (OR: 0.48, CI: 0.32-0.71), Hispanic vs. non-Hispanic (OR: 0.63, CI: 0.41-0.96) children, and those with Medicaid vs. private insurance (OR: 0.7, CI: 0.51-0.97). Mortality was almost double among African American vs. white children (OR: 1.83, CI: 1.03-3.24). Moreover, children in the Northeast and West (vs. South) are more likely to be offered a kidney transplant (90). The goal for the immediate future will be to identify the causes for these health disparities and to begin to address them.

Overall, graft survival and infection-related complications are comparable between transplantation patients with LN-associated ESKD and allograft recipients with ESKD because of other causes $(90,91)$. Serological markers of disease activity (complement C3 and C4 levels, dsDNA antibodies) are even less accurate during the post-transplantation period. Fortunately, recurrent nephritis is very low: less than $3 \%$ of the patients had symptomatic disease. Only $7 \%$ of graft failures are attributable to recurrent LN. However, if a patient has recurrent nephritis, they have a fourfold increased risk for graft failure.

\section{CONCLUSION}

cSLE is an extremely complex disease that has been difficult to diagnose and treat. Given this complexity, pediatricians and pediatric subspecialists treating these patients have depended on astute clinical observations. There remains a great need for more research in children with LN to develop a more precise classification system of kidney injury based on more intricate 
molecular details of cellular function and inflammation, with emerging technologies offering new tools to address this gap in scientific knowledge. Besides basic and translational research in cSLE, robust epidemiological information is needed to focus research and resources appropriately in support of improved prognosis in cSLE. Repeat kidney biopsies in patients followed in prospective registries will more information on natural history. Real-time bedside and home monitoring technology will reduce the time to diagnosis of SLE flares, exacerbations, and treatment failures. Social media and novel approaches to health care provision may increase the accuracy with which patient outcomes can be measured, medication side effects recorded, and ultimately treatment adherence monitored. If the progress made in the past $50 \mathrm{y}$ can be matched over the next $50 \mathrm{y}$, then surely our patients will benefit.

\section{STATEMENT OF FINANCIAL SUPPORT}

None.

Disclosure: The authors do not have any potential conflicts of interest or financial ties to disclose.

\section{REFERENCES}

1. Silva CA, Avcin T, Brunner HI. Taxonomy for systemic lupus erythematosus with onset before adulthood. Arthritis Care Res (Hoboken) 2012;64:1787-93.

2. Livingston B, Bonner A, Pope J. Differences in clinical manifestations between childhood-onset lupus and adult-onset lupus: a meta-analysis. Lupus 2011;20:1345-55.

3. Hiraki LT, Feldman CH, Liu J, et al. Prevalence, incidence, and demographics of systemic lupus erythematosus and lupus nephritis from 2000 to 2004 among children in the US Medicaid beneficiary population. Arthritis Rheum 2012;64:2669-76.

4. Ambrose N, Morgan TA, Galloway J, Ionnoau Y, Beresford MW, Isenberg DA. Differences in disease phenotype and severity in SLE across age groups. Lupus 2016;25(14):1542-50.

5. Brunner HI, Gladman DD, Ibanez D, Urowitz MD, Silverman ED. Difference in disease features between childhood-onset and adult-onset systemic lupus erythematosus. Arthritis Rheum 2008;58:556-62.

6. Hagge WW, Burke EC, Stickler GB. Treatment of systemic lupus erythematosus complicated by nephritis in children. Pediatrics 1967;40:822-7.

7. Meislin AG, Rothfield N. Systemic lupus erythematosus in childhood. Analysis of 42 cases, with comparative data on 200 adult cases followed concurrently. Pediatrics 1968;42:37-49.

8. Caeiro F, Michielson FM, Bernstein R, Hughes GR, Ansell BM. Systemic lupus erythematosus in childhood. Ann Rheum Dis 1981;40:325-31.

9. Hargraves MM. Production in vitro of the L.E. cell phenomenon; use of normal bone marrow elements and blood plasma from patients with acute disseminated lupus erythematosus. Proc Staff Meet Mayo Clin 1949;24:234-7.

10. Hochberg MC. Updating the American College of Rheumatology revised criteria for the classification of systemic lupus erythematosus. Arthritis Rheum 1997;40:1725.

11. Tarr T, Dérfalvi B, Győri N, et al. Similarities and differences between pediatric and adult patients with systemic lupus erythematosus. Lupus 2015;24:796-803.

12. Petri M, Orbai AM, Alarcón GS, et al. Derivation and validation of the Systemic Lupus International Collaborating Clinics classification criteria for systemic lupus erythematosus. Arthritis Rheum 2012;64:2677-86.

13. Sag E, Tartaglione A, Batu ED, et al. Performance of the new SLICC classification criteria in childhood systemic lupus erythematosus: a multicentre study. Clin Exp Rheumatol 2014;32:440-4.

14. Fonseca AR, Gaspar-Elsas MI, Land MG, de Oliveira SK. Comparison between three systems of classification criteria in juvenile systemic lupus erythematous. Rheumatology (Oxford) 2015;54:241-7.
15. Sinha MD, Lewis MA, Bradbury MG, Webb NJ. Percutaneous real-time ultrasound-guided renal biopsy by automated biopsy gun in children: safety and complications. J Nephrol 2006;19:41-4.

16. Tøndel C, Vikse BE, Bostad L, Svarstad E. Safety and complications of percutaneous kidney biopsies in 715 children and 8573 adults in Norway 1988-2010. Clin J Am Soc Nephrol 2012;7:1591-7.

17. Weening JJ, D’Agati VD, Schwartz MM, et al. The classification of glomerulonephritis in systemic lupus erythematosus revisited. J Am Soc Nephrol 2004;15:241-50.

18. Corwin HL, Schwartz MM, Lewis EJ. The importance of sample size in the interpretation of the renal biopsy. Am J Nephrol 1988;8:85-9.

19. Boneparth A, Ilowite NT; CARRA Registry Investigators. Comparison of renal response parameters for juvenile membranous plus proliferative lupus nephritis versus isolated proliferative lupus nephritis: a cross-sectional analysis of the CARRA Registry. Lupus 2014;23:898-904.

20. Hagelberg S, Lee Y, Bargman J, et al. Longterm followup of childhood lupus nephritis. J Rheumatol 2002;29:2635-42.

21. Zappitelli M, Duffy C, Bernard C, et al. Clinicopathological study of the WHO classification in childhood lupus nephritis. Pediatr Nephrol 2004;19:503-10.

22. Rianthavorn P, Buddhasri A. Long-term renal outcomes of childhoodonset global and segmental diffuse proliferative lupus nephritis. Pediatr Nephrol 2015;30:1969-76.

23. Marks SD, Sebire NJ, Pilkington C, Tullus K. Clinicopathological correlations of paediatric lupus nephritis. Pediatr Nephrol 2007;22:77-83.

24. Austin HA 3rd, Muenz LR, Joyce KM, et al. Prognostic factors in lupus nephritis. Contribution of renal histologic data. Am J Med 1983;75:382-91.

25. Hill GS, Delahousse M, Nochy D, et al. A new morphologic index for the evaluation of renal biopsies in lupus nephritis. Kidney Int 2000;58: 1160-73.

26. Zappitelli M, Duffy CM, Bernard C, Gupta IR. Evaluation of activity, chronicity and tubulointerstitial indices for childhood lupus nephritis. Pediatr Nephrol 2008;23:83-91.

27. Brunner HI, Bennett MR, Abulaban K, et al. Development of a novel renal activity index of lupus nephritis in children and young adults. Arthritis Care Res (Hoboken) 2016;68:1003-11.

28. Wenderfer SE, Lane JC, Shatat IF, von Scheven E, Ruth NM. Practice patterns and approach to kidney biopsy in lupus: a collaboration of the Midwest Pediatric Nephrology Consortium and the Childhood Arthritis and Rheumatology Research Alliance. Pediatr Rheumatol Online J 2015;13:26.

29. Hinze CH, Suzuki M, Klein-Gitelman M, et al. Neutrophil gelatinase-associated lipocalin is a predictor of the course of global and renal childhoodonset systemic lupus erythematosus disease activity. Arthritis Rheum 2009;60:2772-81.

30. Suzuki M, Wiers K, Brooks EB, et al. Initial validation of a novel protein biomarker panel for active pediatric lupus nephritis. Pediatr Res 2009;65:530-6.

31. Watson L, Tullus K, Pilkington C, et al. Urine biomarkers for monitoring juvenile lupus nephritis: a prospective longitudinal study. Pediatr Nephrol 2014;29:397-405.

32. Rubinstein T, Pitashny M, Levine B, et al. Urinary neutrophil gelatinaseassociated lipocalin as a novel biomarker for disease activity in lupus nephritis. Rheumatology (Oxford) 2010;49:960-71.

33. Rubinstein TB, Putterman C, Goilav B. Biomarkers for CNS involvement in pediatric lupus. Biomark Med 2015;9:545-58.

34. Abulaban KM, Fall N, Nunna R, et al. Relationship of cell-free urine MicroRNA with lupus nephritis in children. Pediatr Rheumatol Online J 2016;14:4.

35. Sule SD, Moodalbail DG, Burnham J, Fivush B, Furth SL. Predictors of kidney disease in a cohort of pediatric patients with lupus. Lupus 2015;24: $862-8$.

36. Mina R, von Scheven E, Ardoin SP, et al.; Carra SLE Subcommittee. Consensus treatment plans for induction therapy of newly diagnosed proliferative lupus nephritis in juvenile systemic lupus erythematosus. Arthritis Care Res (Hoboken) 2012;64:375-83.

37. Kavanaugh AF, Solomon DH; American College of Rheumatology Ad Hoc Committee on Immunologic Testing Guidelines. Guidelines for immu- 
nologic laboratory testing in the rheumatic diseases: anti-DNA antibody tests. Arthritis Rheum 2002;47:546-55.

38. Sturfelt G, Truedsson L. Complement and its breakdown products in SLE. Rheumatology (Oxford) 2005;44:1227-32.

39. Henderson L, Masson P, Craig JC, et al. Treatment for lupus nephritis. Cochrane Database Syst Rev 2012;12:CD002922.

40. Barron KS, Person DA, Brewer EJ Jr, Beale MG, Robson AM. Pulse methylprednisolone therapy in diffuse proliferative lupus nephritis. J Pediatr 1982;101:137-41.

41. Lehman TJ, Onel K. Intermittent intravenous cyclophosphamide arrests progression of the renal chronicity index in childhood systemic lupus erythematosus. J Pediatr 2000;136:243-7.

42. Lau KK, Ault BH, Jones DP, Butani L. Induction therapy for pediatric focal proliferative lupus nephritis: cyclophosphamide versus mycophenolate mofetil. J Pediatr Health Care 2008;22:282-8.

43. Silverman ED, Lang B. An overview of the treatment of childhood SLE. Scand J Rheumatol 1997;26:241-6.

44. Houssiau FA, Vasconcelos C, D'Cruz D, et al. The 10-year follow-up data of the Euro-Lupus Nephritis Trial comparing low-dose and high-dose intravenous cyclophosphamide. Ann Rheum Dis 2010;69:61-4.

45. Falcini F, Capannini S, Martini G, et al. Mycophenolate mofetil for the treatment of juvenile onset SLE: a multicenter study. Lupus 2009;18: $139-43$.

46. Kizawa T, Nozawa T, Kikuchi M, et al. Mycophenolate mofetil as maintenance therapy for childhood-onset systemic lupus erythematosus patients with severe lupus nephritis. Mod Rheumatol 2015;25:210-4.

47. Buratti S, Szer IS, Spencer CH, Bartosh S, Reiff A. Mycophenolate mofetil treatment of severe renal disease in pediatric onset systemic lupus erythematosus. J Rheumatol 2001;28:2103-8.

48. Tamirou F, D'Cruz D, Sangle S, et al.; MAINTAIN Nephritis Trial Group. Long-term follow-up of the MAINTAIN Nephritis Trial, comparing azathioprine and mycophenolate mofetil as maintenance therapy of lupus nephritis. Ann Rheum Dis 2016;75:526-31.

49. Chen W, Tang X, Liu Q, et al. Short-term outcomes of induction therapy with tacrolimus versus cyclophosphamide for active lupus nephritis: A multicenter randomized clinical trial. Am J Kidney Dis 2011;57:235-44.

50. Fu LW, Yang LY, Chen WP, Lin CY. Clinical efficacy of cyclosporin a neoral in the treatment of paediatric lupus nephritis with heavy proteinuria. Br J Rheumatol 1998;37:217-21.

51. Aragon E, Resontoc LP, Chan YH, et al. Long-term outcomes with multitargeted immunosuppressive protocol in children with severe proliferative lupus nephritis. Lupus 2016;25:399-406.

52. Pereira T, Abitbol CL, Seeherunvong W, et al. Three decades of progress in treating childhood-onset lupus nephritis. Clin J Am Soc Nephrol 2011;6:2192-9.

53. Willems M, Haddad E, Niaudet P, et al.; French Pediatric-Onset SLE Study Group. Rituximab therapy for childhood-onset systemic lupus erythematosus. J Pediatr 2006;148:623-7.

54. Lehman TJ, Singh C, Ramanathan A, et al. Prolonged improvement of childhood onset systemic lupus erythematosus following systematic administration of rituximab and cyclophosphamide. Pediatr Rheumatol Online J 2014;12:3.

55. Watson L, Beresford MW, Maynes C, et al. The indications, efficacy and adverse events of rituximab in a large cohort of patients with juvenile-onset SLE. Lupus 2015;24:10-7.

56. Tambralli A, Beukelman T, Cron RQ, Stoll ML. Safety and efficacy of rituximab in childhood-onset systemic lupus erythematosus and other rheumatic diseases. J Rheumatol 2015;42:541-6.

57. Gladman D, Ginzler E, Goldsmith C, et al. The development and initial validation of the Systemic Lupus International Collaborating Clinics/ American College of Rheumatology damage index for systemic lupus erythematosus. Arthritis Rheum 1996;39:363-9.

58. Brunner HI, Silverman ED, To T, Bombardier C, Feldman BM. Risk factors for damage in childhood-onset systemic lupus erythematosus: cumulative disease activity and medication use predict disease damage. Arthritis Rheum 2002;46:436-44.

59. Sato JO, Corrente JE, Saad-Magalhães C. Chronic active disease pattern predicts early damage in juvenile systemic lupus erythematosus. Lupus 2015;24:1421-8.
60. Mina R, Klein-Gitelman MS, Nelson S, et al. Validation of the systemic lupus erythematosus responder index for use in juvenile-onset systemic lupus erythematosus. Ann Rheum Dis 2014;73:401-6.

61. Levy DM, Peschken CA, Tucker LB, et al. Health related quality of life in childhood-onset systemic lupus erythematosus is associated with ethnicity: Results from a multiethnic multicenter Canadian cohort. Arthritis Care Res 2014;66:1767-74.

62. Schanberg LE, Sandborg C, Barnhart HX, et al.; Atherosclerosis Prevention in Pediatric Lupus Erythematosus Investigators. Use of atorvastatin in systemic lupus erythematosus in children and adolescents. Arthritis Rheum 2012;64:285-96.

63. Rubin HR, Pronovost P, Diette GB. The advantages and disadvantages of process-based measures of health care quality. Int J Qual Health Care 2001;13:469-74.

64. Hollander MC, Sage JM, Greenler AJ, et al. International consensus for provisions of quality-driven care in childhood-onset systemic lupus erythematosus. Arthritis Care Res (Hoboken) 2013;65:1416-23.

65. Mina R, Harris JG, Klein-Gitelman MS, et al. Initial benchmarking of the quality of medical care in childhood-onset systemic lupus erythematosus. Arthritis Care Res (Hoboken) 2016;68:179-86.

66. Tunnicliffe DJ, Singh-Grewal D, Chaitow J, et al. Lupus means sacrifices: perspectives of adolescents and young adults with systemic lupus erythematosus. Arthritis Care Res (Hoboken) 2016;68:828-37.

67. Brunner HI, Mina R, Pilkington C, et al. Preliminary criteria for global flares in childhood-onset systemic lupus erythematosus. Arthritis Care Res (Hoboken) 2011;63:1213-23.

68. Felsenstein S, Reiff AO, Ramanathan A. Transition of care and healthrelated outcomes in pediatric-onset systemic lupus erythematosus. Arthritis Care Res (Hoboken) 2015;67:1521-8.

69. KDIGOcommunications. KDIGO Clinical Practice Guideline for Glomerulonephritis, 2012. http://kdigo.org/home/glomerulonephritis-gn/.

70. Ruggiero B, Vivarelli M, Gianviti A, et al. Lupus nephritis in children and adolescents: results of the Italian Collaborative Study. Nephrol Dial Transplant 2013;28:1487-96.

71. Levy DM, Peschken CA, Tucker LB, et al. The 1000 Canadian faces of lupus: Influence of ethnicity on disease in the pediatric cohort. Arthritis Care Res 2012;65:152-60.

72. Lewandowski LB, Schanberg LE, Thielman N, et al. Severe disease presentation and poor outcomes among pediatric systemic lupus erythematosus patients in South Africa. Lupus 2016; e-pub ahead of print 3 August 2016.

73. Uziel Y, Gorodnitski N, Mukamel M, et al.; Pediatric Rheumatology Study Group Of Israel @ SLERI. Outcome of a national Israeli cohort of pediatric systemic lupus erythematosus. Lupus 2007;16:142-6.

74. Fish AJ, Blau EB, Westberg NG, Burke BA, Vernier RL, Michael AF. Systemic lupus erythematosus within the first two decades of life. Am J Med 1977;62:99-117.

75. McCurdy DK, Lehman TJ, Bernstein B, et al. Lupus nephritis: prognostic factors in children. Pediatrics 1992;89:240-6.

76. Glidden RS, Mantzouranis EC, Borel Y. Systemic lupus erythematosus in childhood: clinical manifestations and improved survival in fifty-five patients. Clin Immunol Immunopathol 1983;29:196-210.

77. Vyas S, Hidalgo G, Baqi N, Von Gizyki H, Singh A. Outcome in AfricanAmerican children of neuropsychiatric lupus and lupus nephritis. Pediatr Nephrol 2002;17:45-9.

78. Baqi N, Moazami S, Singh A, Ahmad H, Balachandra S, Tejani A. Lupus nephritis in children: a longitudinal study of prognostic factors and therapy. J Am Soc Nephrol 1996;7:924-9.

79. Bogdanović R, Nikolić V, Pasić $S$, et al. Lupus nephritis in childhood: a review of 53 patients followed at a single center. Pediatr Nephrol 2004;19:36-44.

80. Vachvanichsanong P, Dissaneewate P, McNeil E. Twenty-two years' experience with childhood-onset SLE in a developing country: are outcomes similar to developed countries? Arch Dis Child 2011;96:44-9.

81. Batinić D, Milošević $D$, Čorić $M$, Topalović-Grković $M$, Jelušić $M$, Turudić D. Lupus nephritis in Croatian children: clinicopathologic findings and outcome. Lupus 2015;24:307-14.

82. Hersh AO, Trupin L, Yazdany J, et al. Childhood-onset disease as a predictor of mortality in an adult cohort of patients with systemic lupus erythematosus. Arthritis Care Res (Hoboken) 2010;62:1152-9. 
83. Tektonidou MG, Dasgupta A, Ward MM. Risk of end-stage renal disease in patients with lupus nephritis, 1971-2015: a systematic review and bayesian meta-analysis. Arthritis Rheumatol 2016;68:1432-41.

84. Wu JY, Yeh KW, Huang JL. Early predictors of outcomes in pediatric lupus nephritis: focus on proliferative lesions. Semin Arthritis Rheum 2014;43:513-20.

85. Singh S, Abujam B, Gupta A, et al. Childhood lupus nephritis in a developing country-24 years' single-center experience from North India. Lupus 2015;24:641-7.

86. Askenazi D, Myones B, Kamdar A, et al. Outcomes of children with proliferative lupus nephritis: the role of protocol renal biopsy. Pediatr Nephrol 2007;22:981-6.

87. Lee BS, Cho HY, Kim EJ, et al. Clinical outcomes of childhood lupus nephritis: a single center's experience. Pediatr Nephrol 2007;22:222-31.
88. Nathanson S, Salomon R, Ranchin B, et al. Prognosis of lupus membranous nephropathy in children. Pediatr Nephrol 2006;21: 1113-6.

89. Hugle B, Silverman ED, Tyrrell PN, Harvey EA, Hébert D, Benseler SM. Presentation and outcome of paediatric membranous non-proliferative lupus nephritis. Pediatr Nephrol 2015;30:113-21.

90. Hiraki LT, Lu B, Alexander SR, et al. End-stage renal disease due to lupus nephritis among children in the US, 1995-2006. Arthritis Rheum 2011;63:1988-97.

91. Bartosh SM, Fine RN, Sullivan EK. Outcome after transplantation of young patients with systemic lupus erythematosus: a report of the North American pediatric renal transplant cooperative study. Transplantation 2001;72:973-8. 